\title{
IMPROVING RADAR PERFORMANCE WITH CONSTANT ENVELOPE MULTI-LEVEL CHIRP
}

\author{
Farhad BAHADORI-JAHROMI, Alireza HASSANNEJAD
}

\begin{abstract}
M-level Continuous Phase Chirp Modulation is considered for data transmission. In this article, we would like to propose a new approach to transmitting information with the help of multi-level wave guide signaling. The main problem with the classic chirp modulation system is that the final phase of the signal is not equal in each period to the initial phase of the next step, and this discontinuity is present in each data transmission period. The main objective of this paper is to improve the efficiency of chirp modulation, which is recommended for continuous phase multi-level scalar modulation, which results has a significant increase in system performance. In this new approach, for sending the $M$ symbols, we use the $\mathrm{M}$ level to send the message. With the help of this idea, we simulate the telecommunication system and examine the probability of its bit error in the presence of additive white Gaussian noise and Rician fading.
\end{abstract}

Keywords: chirp signal; Multi-level Chirp Modulation; detection; phase modulation

\section{INTRODUCTION}

For the first time, the signal was taken into consideration at Bell phone Labs. The chirp signal is a signal whose frequency varies with time. If the frequency of this signal changes linearly with time, then it is called the linear trick signal or LFM; if its frequency is changed nonlinearly, it is called a non-linear tangle signal or NLFM. Linear jump signal was used for radar applications for the first time and afterwards it was widely used in radar and sonar systems because of its many advantages that have been researched alongside its uses. One of the main advantages of the chirp signal is the lack of full sensitivity to the Doppler shifter, as well as there is a variety of hardware to formulate and process it. In recent years, many studies have been carried out on the use of signal jig in telecommunication applications [1]. One of the most important issues in telephony is pulse shaping, since all the main characteristics of the telecommunications system include detection efficiency, distance separation and class. Initial factors that are effective in choosing pulse shapes include spacing coverage and Doppler, detection probability, resolution and measurement errors [2].

After the introduction of the use of the wave guide signal in telecommunications applications, many discussions have been presented to provide optimal modulation based on the chirp signal and its detection. Since the LFM signal has wide bandwidth, modulation based on the LFM signal is classified as a broad spectrum technique. This extension in the modulation bandwidth based on the screw signal causes this modulation to have advantages over the modulation of ASK and FSK. In 1999, Jassenda and his colleagues conducted a full study on various methods of detecting the wave guide signal in the time-frequency domain [3]. In 2002, Willet presented a new method for detecting a waveform signal based on the Huff transform, which uses the signal-signaling signal distribution, which is one of the frequency distributions of the signal [4]. In 2006 and 2007, Gelman and his colleagues presented two new methods for signal processing, which are based on basic functions that change the instantaneous phase signal of the base in then [5] and [6].
In 2008, a method was presented in [7], which is a combination of papers [3] and [6]. In 2005, Barbo and his colleagues presented a new method for classifying radar targets - in that paper, the fractional Fourier transform was used to improve the classification of sound waves and the results have been compared with Fourier transform results. In 2009, Schulli and his colleagues presented a new method for processing the chirp signal based on the Fourier transform, which used higher order statistics for this method [8]. In 2011, Gironjin and his colleagues introduced a new conversion called Chirp-z conversion, which was used to process SAR data and showed that this conversion is capable of greatly increasing the accuracy of suffering and Doppler at the same time [9]. In 2014, Cristalina and his colleagues changed the detection algorithms for radar applications that are mobile targets and the signal used by the chirp, which can estimate the speed of the targets with higher accuracy and less computational complexity. For that purpose they used the Chirp Scaling Algorithm or the CSA algorithm [10]. Then, Osama introduced a method called a discrete linear pulse transform, which used the compression of the signal and showed that this conversion has two degrees of greater freedom than the Fourier transform, as well as in applications where the combined signal of several components has a very high accuracy [11]. In 2018, the method introduced by Wei Wang and et al. [12].

\section{MODULATED SIGNAL OF MULTI-LEVEL SCREW WITH FIXED FASTENING}

The general relation for the fixed-tiered multi-level screw signal is as follows:

$$
\xi_{i}(t)=\sqrt{\frac{2 E_{s}}{\tau_{s}}} \cos \left[\omega_{c} t+\psi_{i}(t)+\varphi\right] \quad 0 \leq t<\tau_{s}, i=1,2, \ldots, M
$$

Where $E_{s}$ is the symbol's energy, $\tau_{s}$ the period of symbol, $\omega_{c}$ the carrier frequency, $\psi_{i}(t)$ contains the phase information 
and $\phi$ the initial phase of the signal, and also the function $\psi_{i}(t)$ is defined as follows:

$$
\psi_{i}(t)=I_{i} J(t)
$$

So that

$$
I_{i}= \begin{cases}+i & \text { if } i \text { is odd } \\ -(i-1) & \text { if } i \text { is even }\end{cases}
$$

Which indicates that one of the $M$ symbols or input level \pm 1 , $\pm 3, \ldots, \pm(M-1)$ has been applied to the modulator. In addition, the phase signal function is specified as follows:

$$
J(t)= \begin{cases}0 & t \leq 0, t>\tau_{s} \\ 2 \pi \int_{0}^{t} f_{I}(\tau) \mathrm{d} \tau & 0 \leq t \leq \tau_{s} \\ \pi \eta=\pi(h-\omega) & t=\tau_{s}\end{cases}
$$

Which $\pi \eta$ denotes the final phase of the signal at $t=\tau_{s}$ and $f_{I}(t)$ is the instantaneous frequency function, which is defined as follows:

$$
f_{I}(t)= \begin{cases}0 & t \leq 0, t>\tau_{s} \\ \left(\frac{h}{2 \tau_{s}}\right)-\left(\frac{\omega}{\tau_{s}^{2}}\right) t, & 0 \leq t \leq \tau_{s}\end{cases}
$$

In the above relation, the variables $h$ and $w$ are dimensionless, and $h$ represents the peak-to-peak frequency divider, and $w$ represents the frequency change frequency divided by the symbol rate $\left(1 / \tau_{s}\right)$, and since $\mathrm{h}=(\eta+\omega) ;(\eta, \omega)$ are determined as parameters of signal modulation. It should be noted that in the M-surface scrolling modulation system, for each $\pm 1, \pm 3, \ldots, \pm(M-1)$, there is a curved signal with a specific frequency range. For example, for a binary system, an incremental gain signal for level -1 is defined and a trickle signal with decreasing frequency for the +1 level or vice versa. Block diagram of multi-level chirp system is shown in Fig. 1.

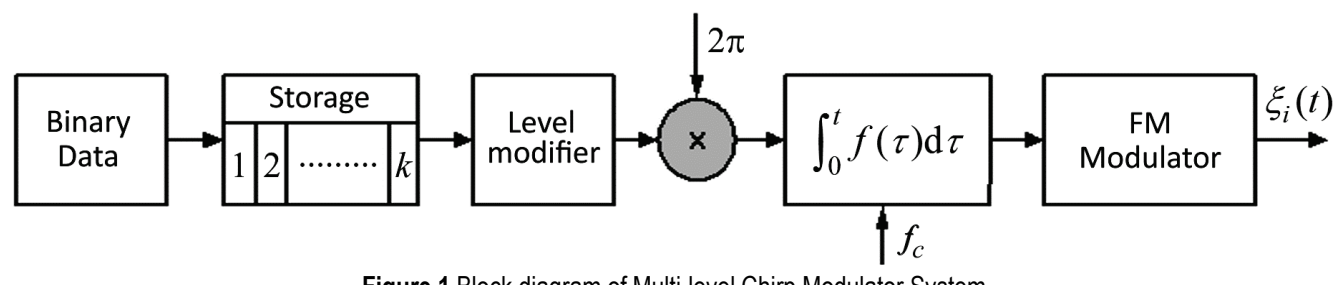

Figure 1 Block diagram of Multi-level Chirp Modulator System

\section{CONTINUOUS PHASE MULTI-LEVEL MODULATION}

The main objective of this paper is to improve the efficiency of chirp modulation, in which continuous multilevel scalar modulation is proposed. This adds a great deal to the system performance, as well as one of the advantages offered by the system and which can be introduced as a memory modulation - in each free space, it must store the initial and final phase information that can model a continuous phase modulation. In addition to introducing the proposed method, the receiver structure for optimal detection is also introduced, and the relationships required for obtaining the probability of error are extracted. The general relation for the continuous phase modulated multi-level wave guide signal is as follows

$\xi(t, I)=\sqrt{\frac{2 E_{s}}{\tau_{s}}} \cos \left[\omega_{c} t+\psi(t, I)+\varphi\right] \quad 0 \leq t<\infty$

Where $E_{s}$ is the symbol's energy, $\tau_{s}$ the symbol period, $\omega_{c}$ the carrier frequency, $\psi_{i}(t, I)$ contains the phase information and $\phi$ the initial phase of the signal, and also the function $\psi_{i}(t, I)$ is defined as follows

$\psi(t, I)=I_{i} J\left[t-(i-1) \tau_{s}\right]+\pi \eta \sum_{k=1}^{i-1} I_{k}, \quad(i-1) \tau_{s} \leq t<i \tau_{s}$
In addition, $I=I_{1}, I_{2}, I_{3}, \ldots$ in such a way that in a noninterpolated sequence a value $d$ is one of the following values

$I_{i}= \pm 1, \pm 3, \ldots, \pm(M-1)$

So that

$P\left(I_{i}\right)=\frac{1}{M} ; i=1,2, \ldots$

In addition, the phase signal function is specified as follows

$J(t)= \begin{cases}0 & t \leq 0, t>\tau_{s} \\ 2 \pi \int_{0}^{t} f_{I}(\tau) \mathrm{d} \tau & 0 \leq t \leq \tau_{s} \\ \pi \eta=\pi(h-\omega) & t=\tau_{s}\end{cases}$

And $f_{I}(t)$ is the instantaneous frequency function that is defined as follows

$f_{I}(t)= \begin{cases}0 & t \leq 0, t>\tau_{s} \\ \left(\frac{h}{2 \tau_{s}}\right)-\left(\frac{\omega}{\tau_{s}^{2}}\right) t, & 0 \leq t \leq \tau_{s}\end{cases}$ 
According to Eq. (7), it is clear that the signal phase value depends not only on the amount of data in the desired symbol, but it is quite obvious that the phase value is dependent on the previous symbols that have entered the modulator (also the memory modulation system). In other words, the first expression is in the fuzzy relation (7), which is determined by the $i^{\text {th }}$ symbol, and the second expression in this relation indicates that the phases associated with the previous symbols are also combined with the obtained phase. Since the signal phase is always a coefficient of $2 \pi$, one can obtain a new path for the phase tree by selecting each parameter $\eta$.

\section{OPTIMAL RECEIVER STRUCTURE}

The detection problem for the signal defined is as follows:

$y(t)=\xi\left(t, I_{\delta}, A\right)+z(t), \quad 0 \leq t \leq n \tau_{s}$

In Eq. (12), $A=\left(I_{1}, I_{2}, \ldots, I_{\delta-1}, I_{\delta+1}, \ldots, I_{n}\right)$ represents one of the $M^{n-1}$ possible sequences of data symbols. Likelihood ratio test is used for detection, so that the correct function for the M-CPCM signal is calculated as follows:

$C F_{M}=\int_{A} \exp \left\{\frac{2}{N_{0}} \int_{0}^{n \tau_{S}} y(t) \xi\left[t, I_{\delta}=-(M-1), A\right] \mathrm{d} t\right\} p(A) \mathrm{d} A$

The distribution of the probability of parameter $A$ is determined as follows:

$p(A)=p\left(I_{1}\right) p\left(I_{2}\right) \ldots p\left(I_{\delta-1}\right) p\left(I_{\delta+1}\right) \ldots p\left(I_{n}\right)$

$p\left(I_{i}\right)=\frac{1}{M}\left\{\delta\left(I_{i}-1\right)+\delta\left(I_{i}+1\right)+\ldots\right.$

$\left.\ldots+\delta\left[I_{i}-(M-1)\right]+\delta\left[I_{i}+(M-1)\right]\right\}$

In addition, the exponential function is obtained as follows

$C F_{M}=1 / m \sum_{k=1}^{m} \exp \left\{\frac{2}{N_{0}} \int_{0}^{n T_{s}} r(t) \xi\left[t, I_{\delta}=-(M-1), A_{k}\right] \mathrm{d} t\right\}$

Finally, the optimal receiver output is calculated by the following equation:

$C F_{k}=\max \left\{C F_{1}, C F_{2}, \ldots, C F_{M}\right\}$

\section{SIMULATION RESULTS}

In Fig. 2, the error rate diagram is shown in terms of the signal-to-noise ratio $\left(N_{\mathrm{b}} / N_{0}\right)$ for the proposed method for the number of different viewing intervals $(n=2,3,4,5)$ for
CPCM-32, as well as the comparison with the modulation of 32PSK and 32FSK.

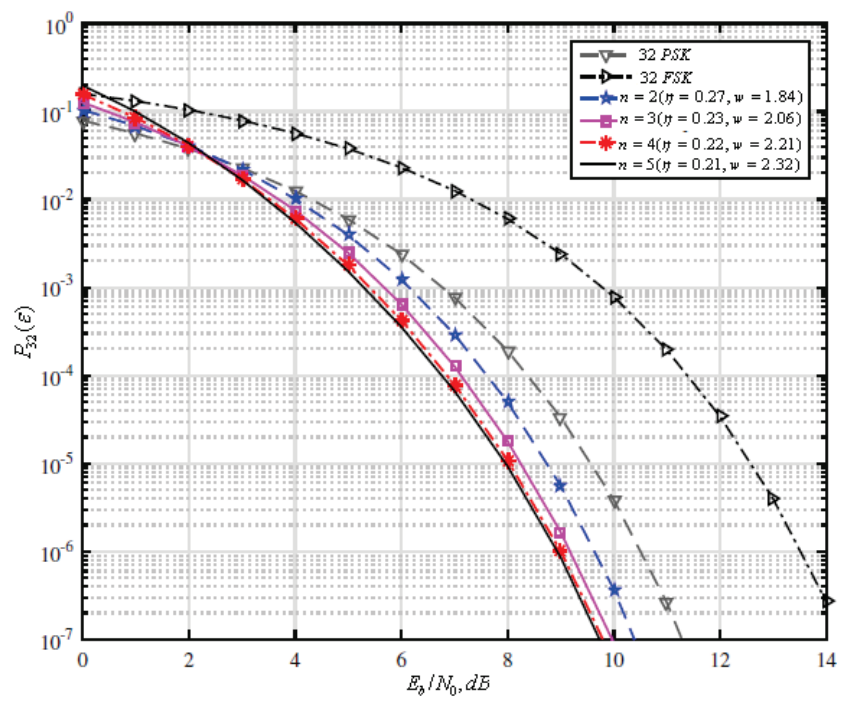

Figure 2 Error probability chart for SNR for system 32-CPCM

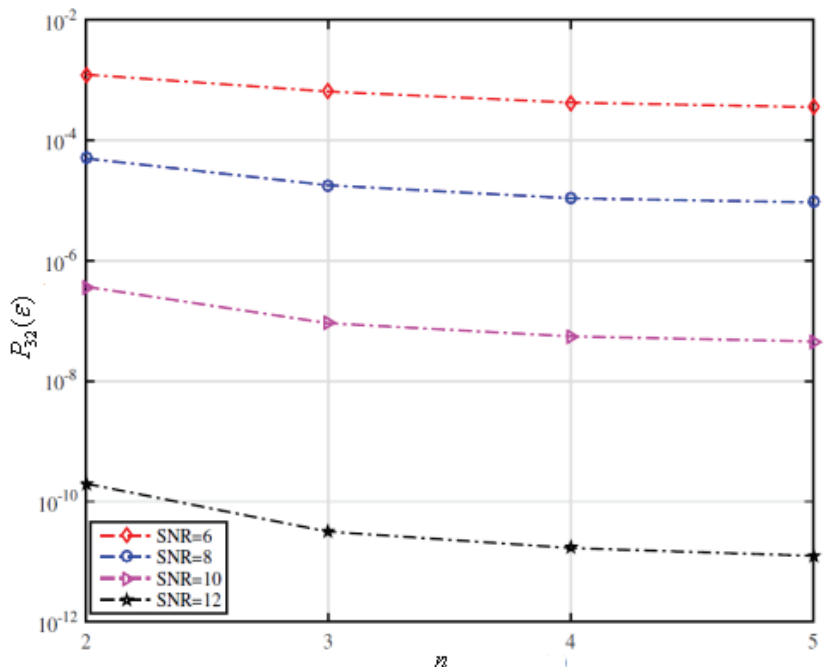

Figure 3 Error probability graph in terms of viewing interval for System 32-CPCM for different SNRs

As shown in Fig. 2, the proposed modulation performance is better than BPSK and FSK. In addition, with the increase in the number of observation intervals, the proposed algorithm improves, and this improvement is negligible with increasing computational complexity due to increasing the value of $\mathrm{n}$. In Fig. 3, we draw the error probability in terms of the observation interval, which we consider to be more precise in reducing the error by increasing the viewing distance.

As shown in Fig. 3, for low SNRs (6 dB and $8 \mathrm{~dB})$, the error rate is reduced slightly with increasing intervals, but for the higher SNRs this improvement (line reduction) is more visible. As $\mathrm{SNR}=12 \mathrm{~dB}$, the error rate is at least less of a 0.01 . On the other hand, by increasing the amount of $n$, the computational volume increases exponentially. 


\section{CONCLUSION}

In this paper, we introduced a new approach for transmitting information using a fixed-tiered multi-level chirp signal, and designed an optimal receiver for its detection. In this new approach, we used the M-symbol to send a different surface. With the help of this idea, we simulate the telecommunication system, and the simulation results show that the proposed modulation function has better performance than classical modulations. In addition, with the increase in the number of observation intervals, the proposed algorithm improves, and this improvement is negligible with increasing computational complexity due to increasing the value of $n$.

\section{REFERENCES}

[1] Alsharef, M., Hamed, A. M., \& Rao, R. K. (2015). Error rate performance of digital chirp communication system over fading channels. Lecture Notes in Engineering and Computer Science: Proceedings of The World Congress on Engineering and Computer Science 2015, 21-23 October, 2015, San Francisco, USA, 727-732.

[2] Khyam, Md. O., Noor-A-Rahim, Md., Li, X., Ritz, C. H., Guan, Y. L., \& Ge, S. S. (2018). Design of Chirp Waveforms for Multiple-access Ultrasonic Indoor Positioning. IEEE Sensors Journal, (99), 1-1, June. https://doi.org/10.1109/JSEN.2018.2846481

[3] Chassande-Mottin, E. \& Flandrin, P. (1999). On the TimeFrequency Detection of Chirps. Applied and Computational Harmonic Analysis, 6, 252-281. https://doi.org/10.1006/acha.1998.0254

[4] Hamed, A. M., Alsharef, M., \& Rao, R. K. (2015). Bit error probability performance bounds of CPFSK over fading channels. Electrical and Computer Engineering (CCECE), 2015 IEEE $28^{\text {th }}$ Canadian Conference on, 1329-1334, 3-6 May. https://doi.org/10.1109/CCECE.2015.7129471

[5] Gelman, L. and Ottley, M. (2006). New processing techniques for transient signals with non-linear variation of the instantaneous frequency in time. Mechanical Systems and Signal Processing, July, p. 1254. https://doi.org/10.1016/j.ymssp.2004.10.002

[6] Alsharef, M. \& Rao, R. K. (2016). Multi-mode multi-level continuous phase chirp modulation: coherent detection. Accepted for Presentation in IEEE CCECE 2016, Vancouver, Canada, 15-18 May. https://doi.org/10.1109/CCECE.2016.7726777

[7] Li, X. \& Bi, G. (2008). A New Transform for Chirp Detection. International Symposium on Information Theory and its Applications, ISITA2008. https://doi.org/10.1109/ISITA.2008.4895439

[8] Qin, Y., Wenyao, L., Shouli, Z., \& Hairong, H. (2009). Detection of Chirp Signal by Combination of Kurtosis Detection and Filtering in Fractional Fourier Domain. Proceedings of the $20092^{\text {nd }}$ International Congress on Image and Signal Processing, CISP'09. https://doi.org/10.1109/CISP.2009.5301566.

[9] Engen, G. \& Larsen, Y. (2011). Efficient Full Aperture Processing of TOPS Mode Data Using the Moving Band Chirp Z-Transform. IEEE Transactions on Geosciences and Remote Sensing, 49(10). https://doi.org/10.1109/TGRS.2011.2145384

[10] Cristallini, D., Pastina, D., Colon, F., \& Lombardo, P. (2012). Efficient Detection and Imaging of Moving Targets in SAR
Images Based on Chirp Scaling. IEEE Transactions on Geoscience and Remote Sensing, 51, 2403-2416. https://doi.org/10.1109/TGRS.2012.2210556

[11] Liu, G.-G., Zhang, L.-R., Liu, N., Chen, G.-F., \& Zhang, Y. (2013). Focusing Highly Squinted Using the Extended Nonlinear Chirp Scaling Algorithm. IEEE Geosciences and Remote Sensing Letters, 10(2), 342-346. https://doi.org/10.1109/LGRS.2012.2203785

[12] Wang, W., Du, J., \& Gao, J. (2018). Multi-Target Detection Method Based on Variable Carrier Frequency Chirp Sequence. Sensors Journal, 8(10), 3386. https://doi.org/10.3390/s18103386

Authors' contacts:

Farhad BAHADORI-JAHROMI,

Department of Electrical and Computer Engineering,

Fasa Branch, Islamic Azad University, Fasa, Iran

E-mail: bahadori.fr@gmail.com

Alireza HASSANNEJAD,

Department of Electrical and Computer Engineering,

Fasa Branch, Islamic Azad University, Fasa, Iran

E-mail: a.hassani9831@yahoo.com 